Conflicts of interest. M.J.G.T.V. has served at the speakers' bureau of Akademie für Infektionsmedizin, Ärztekammer Nordrhein, Astellas Pharma, Basilea, Gilead Sciences, Merck/MSD, Organobalance, and Pfizer, received research funding from 3M, Evoinik, Glycom, Astellas Pharma, DaVolterra, Gilead Sciences, MaaT Pharma, Merck/MSD, Morphochem, Organobalance, and Seres Therapeutics and is a consultant to Alb-Fils Kliniken $\mathrm{GmbH}$, Arderypharm, Astellas Pharma, Ferring, DaVolterra, MaaT Pharma, and Merck/MSD. L.M.B. reports lecture honoraria from Astellas, and MSD and travel grants from $3 \mathrm{M}$ and Gilead. E.S. reports no conflicts of interest relevant to this article.

\section{References}

1. Tomlinson D, Mermel LA, Ethier MC, Matlow A, Gillmeister B, Sung L. Defining bloodstream infections related to central venous catheters in patients with cancer: a systematic review. Clin Infect Dis 2011:697-710.

2. Mermel LA, Farr BM, Sherertz RJ, et al. Guidelines for the management of intravascular catheter-related infections. Clin Infect Dis 2001;32: 1249-1272.

3. Mermel LA, Allon M, Bouza E, et al. Clinical practice guidelines for the diagnosis and management of intravascular catheter-related infection: 2009 update by the Infectious Diseases Society of America. Clin Infect Dis 2009;49:1-45.

4. Fätkenheuer G, Buchheidt D, Cornely OA, et al. Central venous catheter (CVC)-related infections in neutropenic patients-guidelines of the Infectious Diseases Working Party (AGIHO) of the German Society of
Hematology and Oncology (DGHO). Ann Hematol 2003;82 suppl 2: S149-S157.

5. Wolf HH, Leithauser M, Maschmeyer G, et al. Central venous catheterrelated infections in hematology and oncology: guidelines of the Infectious Diseases Working Party (AGIHO) of the German Society of Hematology and Oncology (DGHO). Ann Hematol 2008;87:863-876.

6. Hentrich M, Schalk E, Schmidt-Hieber M, et al. Central venous catheterrelated infections in hematology and oncology: 2012 updated guidelines on diagnosis, management and prevention by the Infectious Diseases Working Party of the German Society of Hematology and Medical Oncology. Ann Oncol 2014;25:936-947.

7. Schalk E, Teschner D, Hentrich M, et al. Central venous catheter-related bloodstream infections in patients with hematological malignancies: comparison of data from a clinical registry and a randomized controlled trial. Infect Control Hosp Epidemiol 2020;41:254-256.

8. Biehl LM, Huth A, Panse J, et al. A randomized trial on chlorhexidine dressings for the prevention of catheter-related bloodstream infections in neutropenic patients. Ann Oncol 2016;27:1916-1922.

9. McHugh ML. Interrater reliability: the kappa statistic. Biochem Med (Zagreb) 2012;22:276-282.

10. Tribler S, Brandt CF, Hvistendahl M, et al. Catheter-related bloodstream infections in adults receiving home parenteral nutrition: substantial differences in incidence comparing a strict microbiological to a clinically based diagnosis. J Parenter Enteral Nutr 2018;42:393-402.

\title{
Regional and statewide antibiograms as targeted interventions against antibiotic resistance
}

\author{
John G Plante BS ${ }^{1}$, Hana R Winders PharmD2 (1), P. Brandon Bookstaver PharmD ${ }^{2,3}$ (D), Majdi N Al-Hasan MBBS ${ }^{1,4}$ (D), \\ Julie Ann Justo PharmD, $\mathrm{MS}^{2,3}$ (D), Katie $\mathrm{S}$ Waites $\mathrm{MPH}^{5}$ and Sharon Weissman MD ${ }^{1,4}$ \\ ${ }^{1}$ University of South Carolina School of Medicine, Columbia, South Carolina, ${ }^{2}$ Department of Clinical Pharmacy and Outcomes Sciences, University of South \\ Carolina College of Pharmacy, Columbia, South Carolina, ${ }^{3}$ Department of Pharmacy, Prisma Health Richland, Columbia, South Carolina, ${ }^{4}$ Department of \\ Medicine, Prisma Health University of South Carolina Medical Group, Columbia, South Carolina and ${ }^{5}$ South Carolina Department of Health and Environmental \\ Control, Columbia, South Carolina
}

To the Editor-Antibiotic resistance is becoming an increasingly heavy burden on our nation, leading to significant patient morbidity, mortality, and healthcare expenditures. ${ }^{1}$ Antibiotic misuse may be considered a primary driver of resistance, and recent studies suggest that $\sim 20 \%-30 \%$ of inpatient antibiotics and $30 \%-40 \%$ of ambulatory antibiotics were inappropriately prescribed. ${ }^{2,3}$ According to the Centers for Disease Control and Prevention's (CDC's) 2019 Threats Report, ${ }^{1}>2.8$ million resistant infections and 35,000 associated deaths are reported annually in the United States. Although this has been accompanied by a $27 \%$ reduction in the number of resistant nosocomial infections, the total number of annual resistant infections has increased, highlighting the need for additional community-focused antimicrobial stewardship interventions. ${ }^{1}$

The impact of several statewide and regional antibiograms on clinical management and stewardship efforts has previously been described. ${ }^{4,5}$ Here, we discuss regional and statewide antibiograms in South Carolina. The Antimicrobial Stewardship Collaborative of

Author for correspondence: Hana R. Winders, Email: hwinders@cop.sc.edu

Cite this article: Plante JG, et al. (2021). Regional and statewide antibiograms as targeted interventions against antibiotic resistance. Infection Control \& Hospital Epidemiology, 42: 503-505, https://doi.org/10.1017/ice.2020.273
South Carolina (ASC-SC) was established in 2016 with support from the CDC. This organization coordinates a variety of statewide antimicrobial stewardship initiatives, including the statewide antibiogram project presented in this letter. This endeavor represents the continuation of a smaller-scale pilot project conducted from 2007 to 2011 through a collaboration between the South Carolina Department of Health and Environmental Control and the University of South Carolina College of Pharmacy.

From 2007 to 2011 and from 2015 to 2017, hospitals and nursing homes throughout South Carolina were asked to submit their annual facility-specific and ambulatory antibiograms to ASC-SC. Each antibiogram was deconstructed into individual isolates and combined into one statewide and multiple regional antibiograms annually. Most of the data consisted of isolates from acute-care hospitals. The yearly cumulative antibiograms were redistributed for use by healthcare facilities across the state.

The compiled statewide antibiogram contains 2017 isolate data from 49 institutions (Fig. 1). Statewide susceptibility rates for the 2017 year were compared to the 2015 year. We used $\chi^{2}$ analysis to assess significance at an $\alpha$ level of 0.05 .

Overall, Escherichia coli was the most frequently reported organism (33,848 isolates in 2017). From 2015 to 2017, Acinetobacter baumannii demonstrated increased susceptibility 
2017 South Carolina Statewide Antibiogram ${ }^{1}$

\begin{tabular}{|c|c|c|c|c|c|c|c|c|c|c|c|c|c|c|c|c|c|c|c|}
\hline Gram Negative Organ isms & 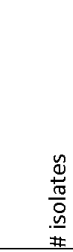 & 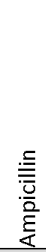 & 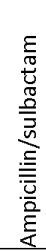 & 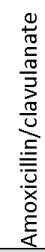 & 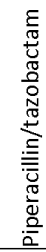 & 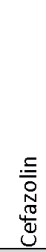 & 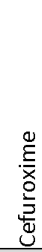 & 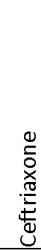 & 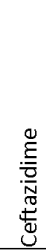 & $\frac{\stackrel{\Xi}{\sigma}}{\frac{\partial}{U}}$ & 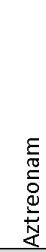 & $\begin{array}{l}\frac{\varepsilon}{d} \\
\frac{\tilde{d}}{\alpha} \\
\frac{0}{0} \\
\frac{0}{\alpha} \\
\Sigma\end{array}$ & 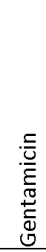 & 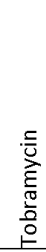 & 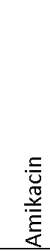 & 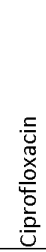 & 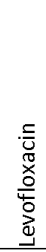 & 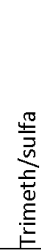 & 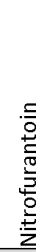 \\
\hline Acinetobacter baumannii & 507 & & 87 & & 79 & & & 47 & 68 & 83 & & 92 & 90 & 95 & 98 & 81 & 83 & 83 & \\
\hline Enterobacter aerogenes $^{2}$ & 996 & & & & 85 & & 50 & 83 & 87 & 98 & 84 & 100 & 99 & 98 & 99 & 96 & 97 & 97 & 19 \\
\hline Enterobacter cloacae & 1930 & & & & 82 & & 42 & 77 & 82 & 94 & 79 & 98 & 95 & 96 & 100 & 90 & 94 & 88 & 35 \\
\hline Escherichia coli & 33848 & 50 & 53 & 83 & 96 & 85 & 89 & 94 & 95 & 95 & 91 & 100 & 90 & 90 & 100 & 75 & 75 & 74 & 95 \\
\hline Klebsiella oxytoca & 621 & & 64 & 86 & 91 & 49 & 86 & 89 & 95 & 93 & 83 & 100 & 95 & 95 & 100 & 95 & 97 & 90 & 78 \\
\hline Klebsiella pneumoniae & 9800 & & 82 & 93 & 93 & 89 & 90 & 94 & 95 & 94 & 89 & 99 & 96 & 94 & 99 & 94 & 94 & 89 & 42 \\
\hline Proteus mirabilis & 5020 & 85 & 94 & 98 & 99 & 90 & 97 & 98 & 98 & 98 & 92 & 100 & 93 & 93 & 100 & 77 & 80 & 81 & \\
\hline Pseudomonas aeruginosa & 5211 & & & & 91 & & & & 88 & 87 & 73 & 92 & 89 & 97 & 96 & 80 & 77 & & \\
\hline
\end{tabular}

\begin{tabular}{|c|c|c|c|c|c|c|c|c|c|c|c|c|c|c|c|}
\hline Gram Positive Organisms & 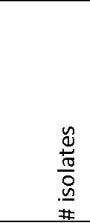 & 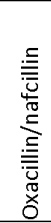 & 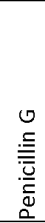 & 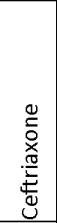 & 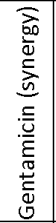 & 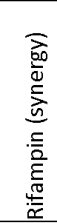 & 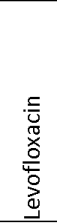 & 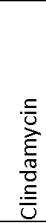 & 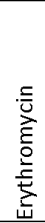 & 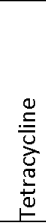 & 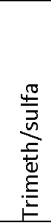 & 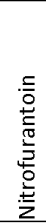 & 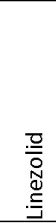 & 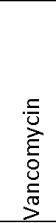 & 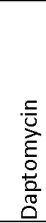 \\
\hline MSSA & 5181 & 100 & 19 & & 97 & 100 & 62 & 77 & 58 & 92 & 99 & 91 & 100 & 100 & 99 \\
\hline MRSA & 6630 & & & & 95 & 96 & 30 & 70 & 13 & 93 & 93 & 84 & 100 & 100 & 99 \\
\hline Total Staphylococcus aureus $^{3}$ & 14647 & 50 & 9 & & 97 & 98 & 52 & 73 & 36 & 93 & 96 & 89 & 100 & 100 & 99 \\
\hline Streptococcus pneumoniae & 609 & & 87 & 93 & & & 99 & 81 & 48 & 78 & 74 & & 100 & 100 & \\
\hline S. pneumoniae (meningitis) & 510 & & 60 & 89 & & & & & & & & & & & \\
\hline
\end{tabular}

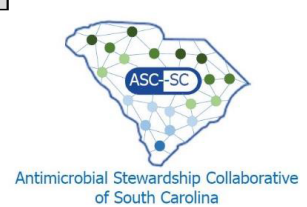

Fig. 1. 2017 South Carolina statewide antibiogram.

${ }^{1}$ This figure contains isolate data from 49 institutions across the state of South Carolina. Numbers represent percent susceptibility. Blank cells correspond to insufficient data or lack of antibiotic testing. Not all reported drugs were tested against every available isolate.

${ }^{2}$ Enterobacter aerogenes is now Klebsiella aerogenes.

${ }^{3}$ Differences between the total number of Staphylococcus aureus isolates versus MSSA plus MRSA isolates are due to variations in facility-specific reporting. Note. Trimeth/sulfa, trimethoprim/sulfamethoxazole; \# isolates, number of isolates; MSSA, methicillin-sensitive Staphylococcus aureus; MRSA, methicillin-resistant Staphylococcus aureus.

to cefepime ( $70 \%$ vs $83 \%, P<.001)$. Susceptibility to ceftazidime and meropenem did not change significantly. Most Enterobacterales exhibited high susceptibility to third- and fourthgeneration cephalosporins, and all were highly susceptible to meropenem. Enterobacter spp proved most resistant, but susceptibility significantly improved for ceftriaxone: E. aerogenes: $78 \%$ versus $83 \%(P=.01)$ and $E$. cloacae: $72 \%$ versus $77 \%$ $(P=.002)$. Despite relatively high resistance rates to levofloxacin and trimethoprim-sulfamethoxazole overall, a slight improvement in susceptibility was observed in E. coli isolates for both levofloxacin $(73 \%$ vs $75 \% ; P<.001)$ and trimethoprim-sulfamethoxazole (73\% vs $74 \% ; P=.01)$.

The susceptibility of Pseudomonas aeruginosa to meropenem increased slightly ( $91 \%$ vs $92 \% ; P=.03$ ) but did not change significantly to cephalosporins or aminoglycosides. The proportion of methicillin-susceptible Staphylococcus aureus increased slightly ( $48 \%$ vs $50 \% ; P=.009$ ). Nonmeningeal Streptococcus pneumoniae exhibited increased susceptibility to penicillin (73\% vs $87 \%$; $P<.001)$ but decreased susceptibility to ceftriaxone ( $96 \%$ vs $93 \%$; $P=.03)$. Susceptibility to erythromycin did not change significantly $(49 \%$ vs $48 \% ; P=.91)$.
A statewide antibiogram may serve as a valuable clinical tool for several reasons. First, an understanding of regional variability in resistance rates could encourage more appropriate empiric antibiotic selection. ${ }^{4,6}$ For example, first-line empiric treatment options for acute pyelonephritis include oral fluoroquinolones, unless community resistance is $>10 \%$, or oral trimethoprimsulfamethoxazole, if the isolate is known to be susceptible. ${ }^{7}$ Our antibiogram demonstrates $E$. coli's poor susceptibility to these agents, and this could guide practitioners, especially in the outpatient setting, to administer a single parenteral dose of a long-acting agent such as ceftriaxone and to more carefully consider patient-specific risk factors for resistance. ${ }^{7-9}$ In patients with acute cystitis, empiric prescribing may be improved by observing the increased susceptibility of nitrofurantoin relative to other oral options.

According to recent guidelines, ${ }^{10}$ macrolides should only be prescribed as empiric monotherapies for community-acquired pneumonia if local resistance to $S$. pneumoniae is known to be $<25 \%$. However, our antibiogram shows minimal organism-specific macrolide susceptibility, thereby encouraging outpatient providers to select a more appropriate empiric treatment (eg, amoxicillin or doxycycline). ${ }^{10}$ 
As illustrated herein, evolving trends in resistance patterns may be easily identified, and this may lead to more targeted, robust infection prevention and control responses. ${ }^{7}$ Individual facilities may also use these data to compare self-reported resistance rates with those in the region. Finally, institutions without access to local antibiograms, including certain outpatient centers and nursing homes, may find this tool especially beneficial to improve prescribing practices and antibiotic stewardship. ${ }^{5}$

Regional and statewide antibiograms have several limiations. Most of our data were gathered from hospitals; relatively few ambulatory antibiograms were submitted. Even though inpatient antibiograms include patients admitted with community-acquired infections, community resistance rates are likely underrepresented. These factors may have led to overestimated community rates, a relevant issue considering the growing concern of resistance in this setting. ${ }^{1,6}$

Despite efforts to standardize antibiogram creation procedures, little evidence supports adherence to these guidelines. Reports therefore undoubtedly vary across institutions, limiting the ability to compare interfacility susceptibility rates. ${ }^{6,7}$ Furthermore, although antibiograms may provide general guidance, other patient-specific factors must be considered to make an informed clinical decision, including the type and severity of the current infection and previous antibiotic use. ${ }^{7}$

In conclusion, statewide and regional antibiograms may be effective strategies in targeting antibiotic resistance. Even though they must be viewed within the scope of their limitations, they should be considered valuable assets in future antibiotic stewardship endeavors.

Acknowledgments. The authors thank advisory board members of the Antimicrobial Stewardship Collaborative of South Carolina for facilitating this study. The findings of this report are those of the authors and do not necessarily represent the official position of the South Carolina Department of Health and Environmental Control.

Financial support. This work was supported by a federal grant (Epidemiology and Laboratory Capacity Project K2) from the Centers for Disease Control and Prevention awarded to South Carolina DHEC.

Conflicts of interest. P.B.B. served as a research advisory board member of Kedrion Biopharma and on the speaker's bureau of bioMérieux. He also received a grant from ALK Abello, Inc. H.R.W. and J.J. also served on the speaker's bureau of bioMérieux. All other authors report no conflicts of interest relevant to this article.

\section{References}

1. US Department of Health and Human Services. Antibiotic resistance threats in the United States, 2019. Centers for Disease Control and Prevention website. https://www.cdc.gov/drugresistance/pdf/threats-report/2019-arthreats-report-508.pdf. Published 2019. Accessed April 18, 2020.

2. Fleming-Dutra KE, Hersh AL, Shapiro DJ, et al. Prevalence of inappropriate antibiotic prescriptions among US ambulatory care visits, 2010-2011. JAMA 2016;315:1864-1873.

3. White AT, Clark CM, Sellick JA, Mergenhagen KA. Antibiotic stewardship targets in the outpatient setting. Am J Infect Control 2019;47:858-863.

4. Leeman H, Zimmermann C, Hansen K, et al. 2017 State antibiogram and implications for antibiotic prescribing. New Hampshire Department of Health and Human Services website. https://www.dhhs.nh.gov/dphs/ cdcs/hai/documents/antibiogram-sum-2017.pdf. Published 2018. Accessed April 18, 2020.

5. Var SK, Hadi R, Khardori NM. Evaluation of regional antibiograms to monitor antimicrobial resistance in Hampton Roads, Virginia. Ann Clin Microbiol Antimicrob 2015;14:22.

6. Nodzo SR, Frisch NB. The use of antibiograms in orthopedic surgery. Curr Rev Musculoskelet Med 2018;11(3):341-346.

7. Gupta K, Hooton TM, Naber KG, et al. International clinical practice guidelines for the treatment of acute uncomplicated cystitis and pyelonephritis in women: a 2010 update by the Infectious Diseases Society of America and the European Society for Microbiology and Infectious Diseases. Clin Infect Dis 2011;52(5):e103-e120.

8. DeMarsh M, Bookstaver PB, Gordon C, et al. Prediction of sulfamethoxazole/trimethoprim resistance in community-onset urinary tract infections. J Glob Antimicrob Resist 2020;21:218-222.

9. Shah A, Justo JA, Bookstaver PB, Kohn J, Albrecht H, Al-Hasan MN. Application of fluoroquinolone resistance score in management of complicated urinary tract infections. Antimicrob Agents Chemother 2017;61(5):e02313-16.

10. Metlay JP, Waterer GW, Long AC, et al. Diagnosis and treatment of adults with community-acquired pneumonia: an official clinical practice guideline of the American Thoracic Society and Infectious Diseases Society of America. Am J Respir Crit Care Med 2019;200(7):e45-e67.

\title{
Zoonotic brucellosis from the long view: Can the past contribute to the present?
}

\author{
Robin Bendrey $\mathrm{PhD}^{1}$ (1) and Guillaume Fournié $\mathrm{PhD}^{2}$ \\ ${ }^{1}$ School of History, Classics and Archaeology, University of Edinburgh, Edinburgh, Scotland and ${ }^{2}$ Veterinary Epidemiology, Economics and Public Health group, \\ Department of Pathobiology and Population Sciences, Royal Veterinary College, University of London, Hatfield, England
}

To the Editor-Zoonotic brucellosis is an endemic disease in many regions of the world, including the Zagros Mountains of Iran and

Author for correspondence: Robin Bendrey, E-mail: robin.bendrey@ed.ac.uk

Cite this article: Bendrey R and Fournié G. (2021). Zoonotic brucellosis from the long view: Can the past contribute to the present?. Infection Control \& Hospital Epidemiology, 42: 505-506, https://doi.org/10.1017/ice.2020.270
Iraq, as Abdi et $\mathrm{al}^{1}$ lay out in a recent article in Infection Control and Hospital Epidemiology. Abdi et al touch upon the history of farming in the Zagros Mountains to contextualize the deep time dimension of brucellosis risk for communities within this region. Building on this, we highlight the context of this early history and the contribution that long-term perspectives of evolving 